\title{
A formalization of one of the main claims of "A linked data model for data scopes" by De Boer et al. 2021'
}

\author{
Victor de Boer \\ Vrije Universiteit Amsterdam, The Netherlands \\ E-mail:v.de.boer@vu.nl; ORCID: https://orcid.org/0000-0001-9079-039X \\ Editor: Cristina-Iulia Bucur (https://orcid.org/0000-0002-7114-6459) \\ Review comments from: Tobias Kuhn (https://orcid.org/0000-0002-1267-0234); Ricardo Usbeck \\ (https://orcid.org/0000-0002-0191-7211); Margherita Martorana (https://orcid.org/0000-0001-8004-0464); Davide Ceolin \\ (https://orcid.org/0000-0002-3357-9130); Mariya Dimitrova (https://orcid.org/0000-0002-8083-6048); Cristina-Iulia Bucur \\ (https://orcid.org/0000-0002-7114-6459)
}

Received 24 June 2021

Accepted 19 November 2021

Abstract. De Boer et al. claimed in previous work that in the context of Digital Humanities research, usage of the Linked Data Scopes ontology contributes to transparency of the research. We present here a formalization of that claim, stating that all things of class "usage of Linked Data Scopes" that are in the context of a thing of class "digital humanities research" can generally have a relation of type "contributes to" to a thing of class "transparency" in the same context.

Keywords: Digital humanities research, usage of linked data scopes, transparency

\section{Introduction}

De Boer et al. [2] state that "With the rise of data driven methods in the humanities, it becomes necessary to develop reusable and consistent methodological patterns for dealing with the various data manipulation steps. This increases transparency, replicability of the research. Data scopes present a qualitative framework for such methodological steps. In this work we present a Linked Data model to represent and share Data Scopes". We present here a formalization of the main scientific claim from this quote by using a semantic template called the super-pattern [1].

\section{Formalization}

Our formalization looks as follows:

\footnotetext{
${ }^{1}$ As RDF/nanopublication: http://purl.org/np/RAgoIxfXPqNDY8vnK2EmBQDAFwuFIDJtfaMplTvPMq3pg 
CONTEXT-CLASS ("in the context of all..."): SUBJECT-CLASS ("things of type..."):

QUALIFIER:

RELATION-TYPE ("have a relation of type..."): OBJECT-CLASS ("to things of type..."): digital humanities research usage of Linked Data Scopes can generally contributes to transparency

In the context class we use a new minted class "digital humanities research" that is a subclass of "research" (Q42240) from Wikidata and is related to the class "digital humanities" (Q1026962) from Wikidata. In the subject class, we use a new minted class "usage of Linked Data Scopes" that is defined using the restriction "some values from" from OWL on "the Data Scopes Ontology" with the property "uses" (P2283) from Wikidata. In the object class we use the class "transparency" (Q535347) from Wikidata.

\section{RDF code}

This is our formalization as a nanopublication in TriG format:

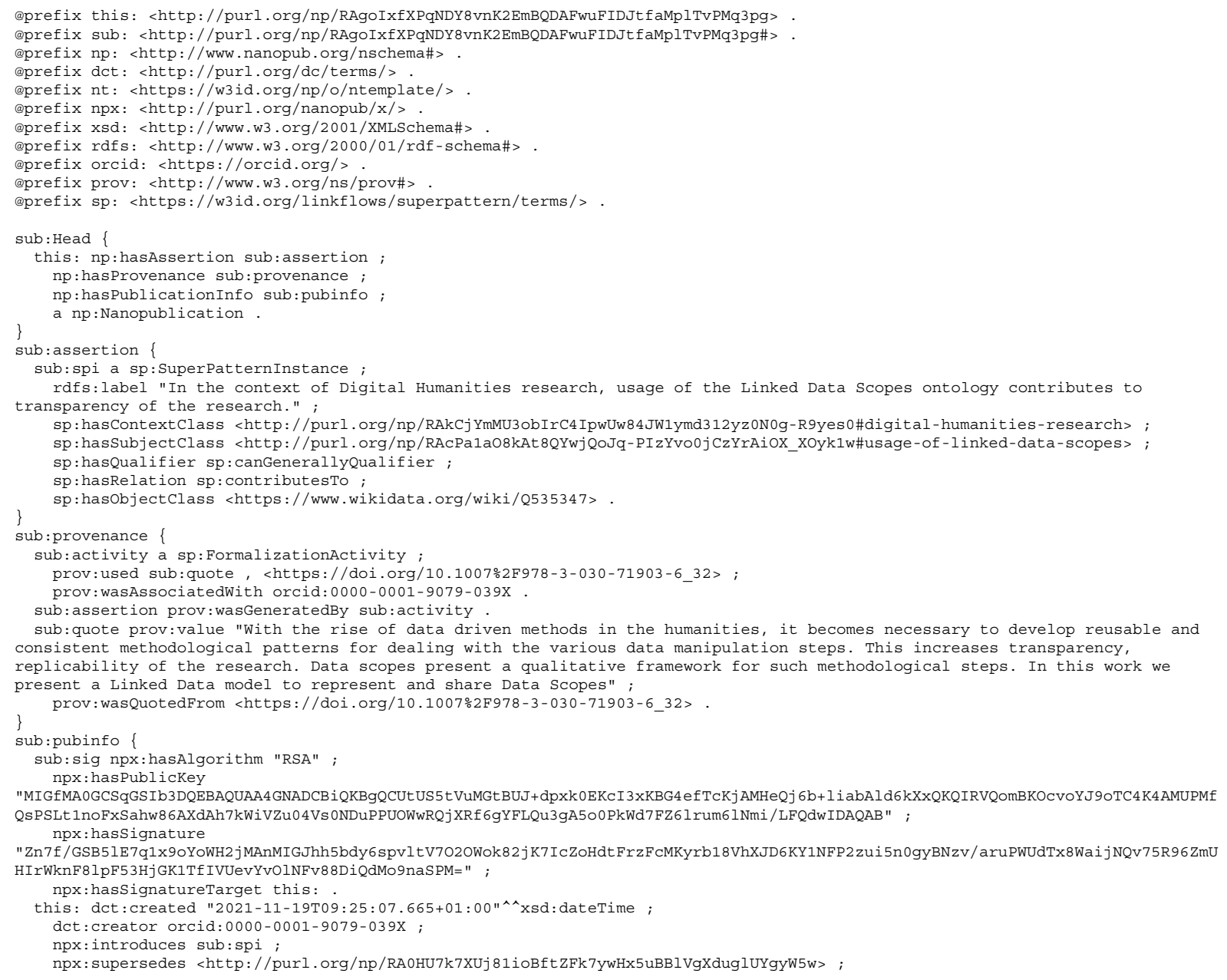


<https://w3id.org/linkflows/reviews/isUpdateOf> <http://purl.org/np/RATc909tCdQPlrsJeGNsTNO0PbENI zdKa6XAOex7dXfrY> ; nt : wasCreatedFromProvenanceTemplate <http://purl.org/np/RAB_oy10D3XUP-zYlqGz7Uj 58AsUXhEKeGqmRFg5LSgDM> nt: wasCreatedFromPubinfoTemplate <http://purl.org/np/RA2VCBẌZf-icECVRGhulJXugTGxpsV5YVr9yqCI1bQh4A> $<$ http://purl.org/np/RAA2MfqdBCzmz9yVWjKLXNbyfBNCwSMmOqCNUxkk1maIM> <http://purl.org/np/RAjpBMlw3 OwYhJUBo3Dt suDIXsNAJ8cnGeWAutDVjuAuI > ; \} nt: wasCreatedFromTemplate <http://purl.org/np/RAv68imZrEjfcp2rnEg1hzoBqEVc0cQMtp9_1Za0BxNM4>

The following nanopublications introduce the newly minted classes in TriG format. This is the class definition of "digital humanities research":

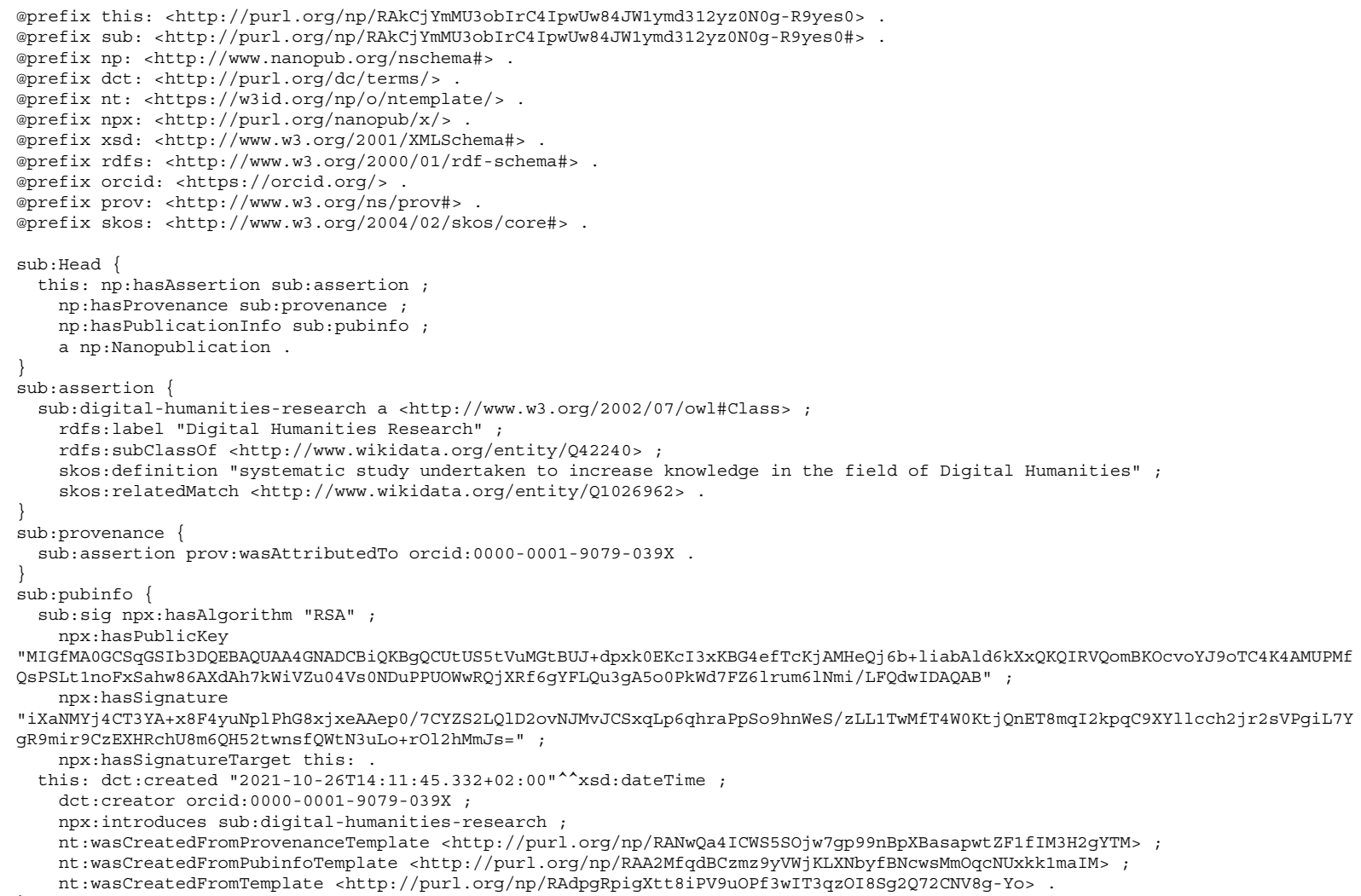

\section{This is the class definition of "usage of Linked Data Scopes":}

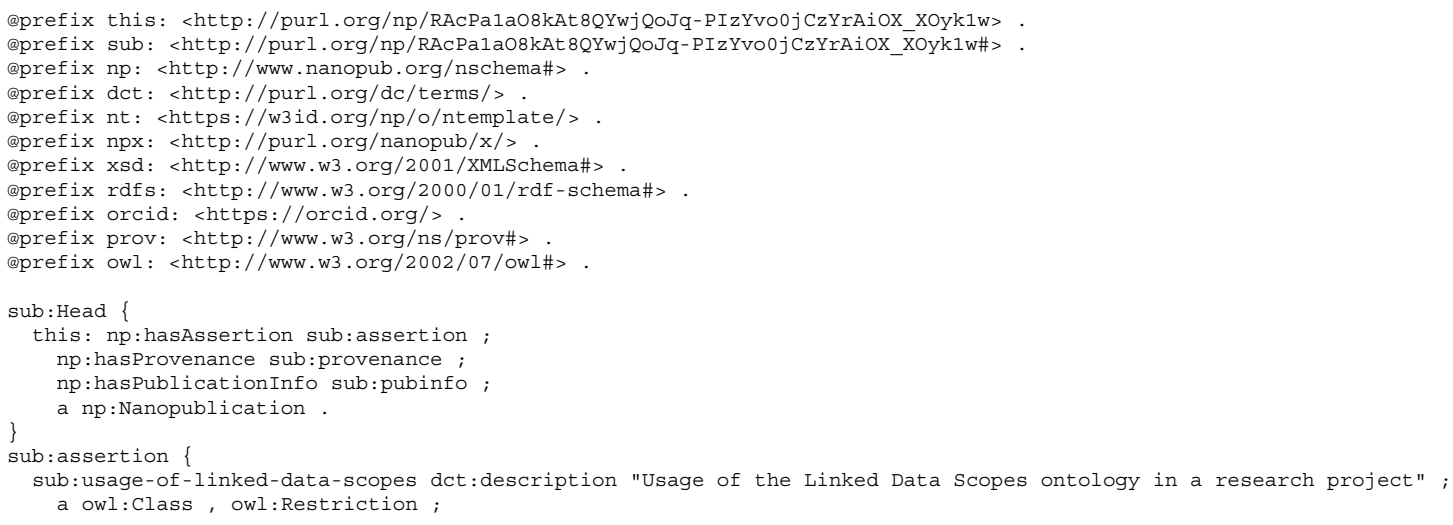




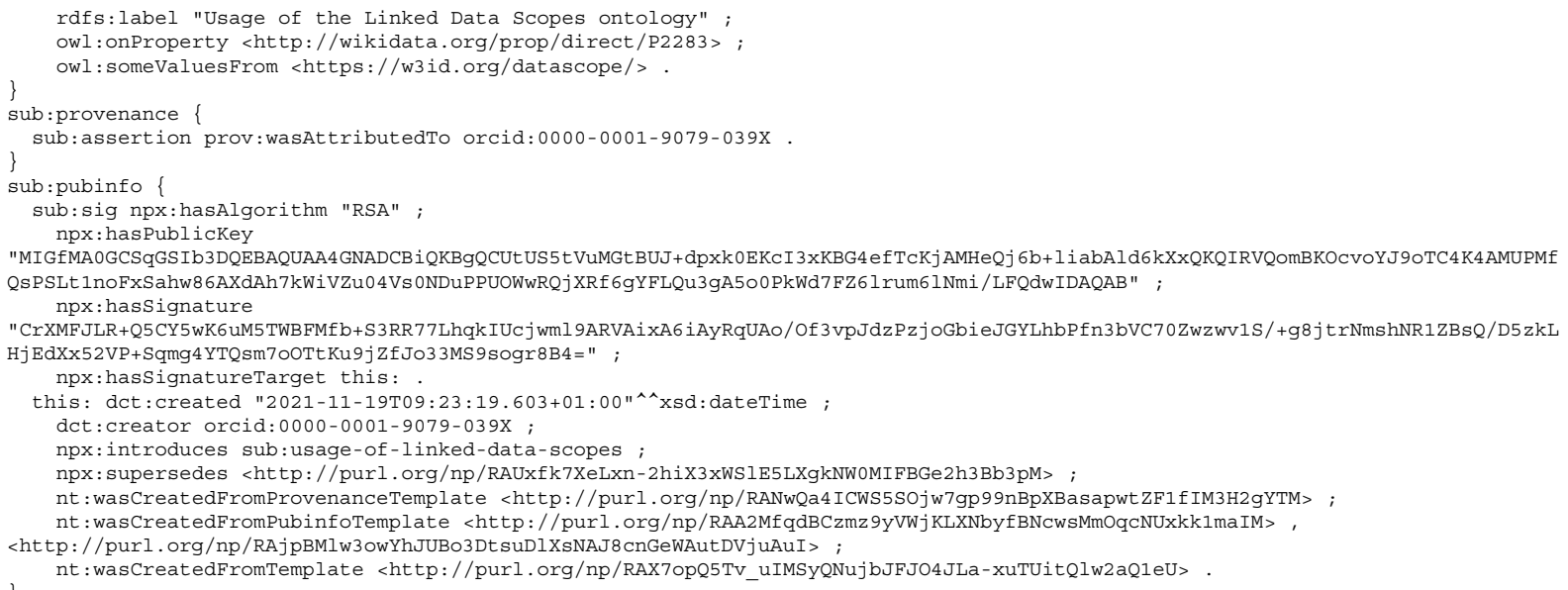

\section{References}

[1] C.I. Bucur, T. Kuhn, D. Ceolin and J. van Ossenbruggen, Expressing high-level scientific claims with formal semantics, in: Proceedings of the 11th Knowledge Capture Conference, 2021. doi:10.1145/3460210.3493561.

[2] V. de Boer, I. Bonestroo, M. Koolen and R. Hoekstra, A linked data model for data scopes, in: Metadata and Semantic Research. MTSR 2020, E. Garoufallou and M.A. Ovalle-Perandones, eds, Communications in Computer and Information Science, Vol. 1355, Springer, Cham, 2021. doi:10.1007/978-3-030-71903-6_32. 